\title{
Reading Between the Lines: Accessing Information via YouTube's Automatic Captioning
}

\author{
Chad Smith and Tamby Allman \\ Texas Woman's University \\ Samantha Crocker \\ Weatherford Regional Day School Program for the Deaf
}

\begin{abstract}
This study and discussion center upon the use of YouTube's automatic captioning feature with college-age adult readers. The study required 75 participants with college experience to view brief middle school science videos with automatic captioning on YouTube and answer comprehension questions based on material presented auditorily and/or through the automatic captions. Participants were divided into groups and presented with the captioned videos with or without sound. The videos, which all focused on the solar system, contained low and high instances of errors within the captions. The research found that comprehension of the automatic caption text varied significantly based on how the participants viewed the videos, with significantly more errors in comprehension for the group that viewed the high error video with automatic captioning only.
\end{abstract}

Keywords: captioning, access, accessibility, online learning, distance education, deaf, hard of hearing

Smith, C., Crocker, S. \& Allman, T. (2017) Reading between the lines: Accessing information via YouTube’s automatic captioning, Online Learning 21(1), 115-131. doi: 10.24059/olj.v21i1.823

\section{Introduction}

Since Google's acquisition of YouTube in 2006, both web giants have been working on developing a captioning method to make web-based video content accessible for deaf and hard of hearing users. YouTube currently offers users who post videos the option-which YouTube strongly encourages - to add subtitles and captions to their video. https://support.google.com/youtube/answer/2734796?rd=1 (3 Play Media, 2014). Also available to users is an automatic captioning feature. The automatic captioning feature is based on speechrecognition technology that employs a complex statistical model for the probability of specific sounds, words, and word combinations occurring within a language. According to Google's YouTube Help site, automatic captioning is available in 10 languages worldwide (Google, 2015). 
While attending class, and preparing university courses that include students who are deaf and/or hard of hearing, the authors noted the volume of errors in the automatic captioning present in several of the videos posted and viewed in YouTube. After some discussion, the authors decided to conduct a preliminary investigation into the overall effectiveness of the automatic captioning tool. To determine the consistency of YouTube's automatic captioning feature of online videos, 50 videos targeted at a middle-school audience were viewed. In each of the videos, a variety of errors and error types were documented. Though some error types were more prominent than others, each error type plays a role in the overall comprehension of the video content. Errors were divided into 11 categories during the review and included addition of words, deletion of words, coherent miscues, incoherent miscues, spelling, incomprehensible phrases, word condensing, homonyms, approximation (content errors), speed, and visual readability. Videos with audio of 1) non-native English speakers with accents, 2) young children's voices, 3) voices that contained mumbling or computer-generated/mechanical sounds were harder to understand and had more captioning errors. The speed of the videos and timing of the captioned content also proved problematic. Readability of the captioned content and aesthetics of the captioning were also noted for each video.

The results of the initial project were gathered by watching the first two minutes of the 50 videos focusing on the solar system with the automatic captioning feature enabled and with content from the $8^{\text {th }}$ grade Texas Essential Knowledge and Skills (TEKS) goals. The frequency and type of errors were documented along with the quality, speed, and type of video. The data were then put into an Excel spreadsheet in preparation for the data analysis. The errors that were documented from each phrase were categorized as follows: additions, deletions, coherent miscues (full-phrase), incoherent miscues (full-phrase), miscues of a single word, word condensing, homonyms, approximations, and morphemes. These categories were established to set up guidelines for what was to be considered an error. This initial evaluation of YouTube's content prompted the current study.

\section{Effects of Captioning}

\section{Review of Literature}

The discussion of captioning audio-visual material must focus on more than simply attempting to present textual representations of audio content on the video. Simply putting text on the screen is insufficient for providing equitable access to the audio content. Successful captioning has been an appropriate supplement to video-based materials and has even been shown to be useful as a foreign language instructional tool when used with videos containing native speaker accents (Dabhi, 2004). Captioning in different formats, including keyword captioning, in which students view the video with partial captioning using only pre-selected keywords while listening to a video at the same time, has proven effective with users of videobased content, especially when the complexity of the video content is beyond the reading level of the viewer (Ruan, 2015). In such instances, the captioned content can help clarify the viewer's understanding of the video content presented. Lewis \& Jackson (2001) found that the script comprehension for captioned videos for students who were deaf or hard of hearing was greater than the comprehension of script in other text forms and increased comprehension beyond the identified reading levels of students. Verbatim captions that are paced to the natural rate of delivery provide access to complete conversational exchanges including both the audio and visual information and allow viewers to comprehend both explicit and implicit information. 
There is an advantage to both deaf and hearing students in terms of comprehension when video and captions are presented (Lewis \& Jackson, 2001). Advantages of captioned video include facilitating novel vocabulary identification and overall comprehension (Winke, Gass, \& Sydorenko, 2010). For second language learners, captioning aids in form-mapping, the process of connecting spoken and written vocabulary, by not having to focus auditorily on word meaning, and instead focus on printed form to connect it with meaning.

Gass, \& Sydorenko, 2010). Information presented verbally and visually is integrated as it is stored in memory (Sadoski \& Paivo, 2004). Johnson-Glenberg (2000) reports that the recall of the linguistic information will stimulate retrieval of the visual information and vice versa. However, even when captioned material contains enhanced or expanded content, it often goes unused by educators despite feedback from students indicating that using captioning while viewing video content would be appealing (Steinson \& Stevenson, 2015).

\section{Successful Caption Use}

There are a number of issues involved with successful captioning of audio-video content. Two issues affecting the overall captioned experience include speed and formatting. Jensema \& McCann (1995) found that the "safe speed" for word content displayed in captioned material was approximately 120-140 words per minute. Unfortunately, captioned material can be presented at speeds exceeding 200 words per minute.

Formatting can make the message delivery problematic as well. Closed-captioning versus automatic captioning and placement of the text on the video content can also play significant roles in how the captioned content is understood. Closed-captioning involves embedded textual content (by an author/programmer) that is timed to present with the audio content synchronously. Closed captioning can be presented live, as with television programming, or post-production, as with cinema movie content, and is not visible until the user activates their decoding systems and displays the captions on their screens. New television sets and video display monitors sold in the United States must be equipped with built-in caption decoder chips. Schools, colleges, libraries and other recipients of federal financial assistance are required under section 508 of the Rehabilitation Act to make their communication accessible to and usable by persons with disabilities (National Association of the Deaf, 2002). Automatic captioning involves the presentation of textual material based solely on the success of speech-recognition software. The captioned content for automatic captioning is not a permanent component of the video, and often varies in accuracy based on the speech delivery of the audio content.

Despite the numbers of individuals with disabilities using social media options and information and communication technologies (ICTs) today, many individuals still struggle with issues related to accessibility (Seale, Georgeson, Mamas, \& Swaim, 2015; Asuncion, Budd, Fichten, Nguyen, Barile, \& Amsel, 2012; Fichten, Asuncion, \& Scapin, 2014). In response to increased population with hearing loss who use Internet-based technologies and social media, YouTube has created an automatic captioning feature. The feature attempts to approximate textbased representations of the speech audio content within videos through the use of speech recognition software. Sadly, researchers and companies, including Google, have recognized that despite the vast resources of Google and YouTube, the automatic captioning can fail to accurately convey the intended message (Barton, Bradbrook, \& Broome, 2015; Johnson, 2014). 
No current research focusing on the success or comprehension of material with the automatic captioning feature of YouTube was found. This mirrors historical trends regarding the use of captioning. In fact, according to Cambra, Silvestre, and Leal (2009), the study of deaf individuals' use of closed-captioning on television has not been a research priority in the field of deaf education. In a review of the literature of closed-captioning on television, no research on the influence of errors within the captioning was located. As captioning for online content is relatively new, the issue of Web-based captioning has not become a priority in deaf education either.

Successful use of captioned video material requires a significant cognitive load visually even when the captioned material is presented appropriately. Cognitive load can be explained as a complex theory that attempts to quantify the burden that performing a specific task imposes on the cognitive system of a learner (Paas \& van Merriënboer, 1994; Paas, Tuovinen, Tabbers, \& Van Gerven, 2003). In relation to this study, cognitive load occurs when a viewer relying on captioned material to access the content presented auditorily must attempt to access both the captioned content and the video-based content simultaneously. The brain is taxed visually and the two modalities compete for delivering the material to the brain via the same cognitive space. That cognitive competition can add stress to the situation making comprehension more difficult, especially for those with limited reading proficiency.

The primary focus for this study is based in part on the assertion by Cambra, Silvestre, and Leal (2009) that reading comprehension and reading speed influence comprehension of closed-captioned text of video content. Because of the complexity of successfully navigating captioned video material, comprehension of such material requires individuals with successful reading skills. The proposed research seeks to evaluate the comprehensibility of YouTube's automatic captioning feature based on the reading abilities of college-educated adult readers. The research question for this study is, "Can college-level adult readers understand basic concepts from science videos posted on YouTube with auto-captioned text containing errors?”

\section{Participants}

\section{Methodology}

Participation in the study was open to students, staff, and faculty at a doctoral-granting, public university in the southwestern US (Table 1). To locate participants with successful reading skills, participants had to have had some college experience in order to participate in the study. The researchers anticipated that participant ages would vary and range from 18 to 60+ years. All genders of adult students, staff, and faculty at the university were allowed to participate. Study participation was dependent on reading ability.

The study sought to determine whether individuals with college-level reading abilities could understand the printed messages of content delivered via the YouTube automatic captioning feature. Participants were required to have the ability to read Web-based automatic captioning and simple sentences and questions, as well as write their own answers to the questions about the videos.

Participants for the research study were from the university community where the research was conducted. Researchers emailed an invitation to university students, faculty, and staff on the primary campus of the university regarding participation in the study. Participants were invited 
to participate in the study using one of the on-campus computer classrooms on the university campus. Participants completed a consent form before participation was permitted.

The research team hosted and monitored the study participants in a university computer classroom/lab using an Internet-connected computer and paper questionnaire. Demographic information was collected at the beginning of the classroom portion of the research (Table 1). The vast majority of participants were female (94\%), with English as a first language (81.2\%), and were hearing (95\%). Only three individuals self-identified as deaf or hard of hearing. Participants watched one of three middle-school videos about the solar system and answered basic questions about the material presented via one of the three viewing options. Using a pen and a paper questionnaire, participants answered a series of questions for one of three video options on the solar system. The video selection, caption availability, and sound availability were chosen at random for each participant and the sound was turned off or on accordingly. The research occurred over a two-week period. Participants were given the option of selecting a date they wished to participate. Participants did not know which video or under which viewing conditions they would be watching the video until they sat down at the computer. Each of the computer stations was randomly set to view one of the videos under a specific viewing option. The questionnaires were numbered to indicate which video and viewing condition. The computers were set up to view the corresponding video under the specific viewing condition. Students self-selected where they sat upon entering the classroom.

Originally, participants were to view one of three middle school science videos on the solar system labeled as having captions with "few" errors, "moderate” errors, or "high" errors. The error types used in determining the groupings of "few-error, moderate-error, and high-error" status were identical to those used at the initial stages of the project to determine the types of errors present in the automatic captioning. Videos were selected from the initial evaluation of 50 videos. Videos were categorized into one of the three groups, based on the number of errors present. To select the videos for the study, the authors used the videos with the highest, median, and lowest numbers of errors. Videos were then re-evaluated to determine which in each group contained standard American English speech from a human (not digitized or robotic speech) with limited to no accents so that the only influence on the automatic captioning was the quality of the speech recognition software. Due to limited participation, the authors focused the initial viewing sessions on the videos with "few" and "high" errors. No participants viewed the videos with "moderate" instances of errors. Participants for each session viewed the videos similarly under one of the following conditions: 1) sound without captioning, 2) sound with automatic captioning, or 3) automatic captioning without sound (Appendix A). Determination of which participant sessions were given a particular viewing method were determined by random selection. Participants who seated themselves at computers where the sound was enabled for the video viewing were provided a new pair of earphones to use throughout the experience. Participants were free to take the earphones with them once their participation was complete. Sessions were arranged so that approximately the same number of participants viewed each video option.

Each of the YouTube videos were watched online during each viewing. The Web addresses for the videos are listed at the end of this document and in the attached video questionnaires (Appendix B). Participants viewed the pre-determined video on a university computer. Participants were free to watch the video and answer the questions as they wished. They could watch the video entirely and then go back and answer questions or they could answer 
questions while watching the video. While participants were only allowed to participate one time in the study, there were no limitations on the number of times they could watch the video or pause and go back during that participation. The researchers had the YouTube site open to the appropriate video upon participants' arrival to the computer lab.

\section{Participants}

\section{Results}

Frequencies and percentages for the demographic variables are displayed in Table 1 . The largest percentage of participants were in group 2 (20.3\%) and the majority of participants were in low error groups (52.7\%). In addition, the largest percentage of participants were in the sound and caption group (35.1\%). Finally, the majority of participants were female (94.0\%), reported that English was their first language (81.2\%), and had identified themselves as hearing (95.1\%).

Means and standard deviations for the continuous variables are displayed in Table 2.

Participants' ages ranged from 18 to $59(M=22.55, S D=6.14)$ and the number of years of college experience ranged from $0<1$ to $11(M=3.21, S D=1.83)$. The number of correct responses by participants ranged from 1 to $12(M=8.76, S D=2.88)$

Table 1. Frequencies and Percentages of Categorical Demographic Variables

\begin{tabular}{|c|c|c|c|}
\hline & & $\mathrm{n}$ & $\%$ \\
\hline \multicolumn{4}{|l|}{ Total Groups } \\
\hline & Group 1 (Low Error, Sound No Caption) & 11 & 14.9 \\
\hline & Group 2 (Low Error, Sound and Caption) & 15 & 20.3 \\
\hline & Group 3 (Low Error, Caption No Sound) & 13 & 17.6 \\
\hline & Group 7 (High Error, Sound No Caption) & 13 & 17.6 \\
\hline & Group 8 (High Error, Sound and Caption) & 11 & 14.9 \\
\hline & Group 9 (High Error, Sound and Caption) & 11 & 14.9 \\
\hline \multicolumn{4}{|l|}{ Error Groups } \\
\hline & Low Error & 39 & 52.7 \\
\hline & High Error & 35 & 47.3 \\
\hline
\end{tabular}




\section{Caption Group}

$\begin{array}{lcc}\text { Sound No Caption } & 24 & 32.4 \\ \text { Sound and Caption } & 26 & 35.1 \\ \text { Caption No Sound } & 24 & 32.4\end{array}$

Gender

$\begin{array}{lcc}\text { Female } & 63 & 94.0 \\ \text { Male } & 3 & 4.50 \\ \text { Transgendered } & 1 & 1.50\end{array}$

English as First Language

No

$13 \quad 18.8$

$\begin{array}{lll}\text { Yes } & 56 & 81.2\end{array}$

Hearing Status

$\begin{array}{lrr}\text { Deaf } & 1 & 1.6 \\ \text { Hard of Hearing } & 2 & 3.3 \\ \text { Hearing } & 58 & 95.1\end{array}$

Table 2. Means and Standard Deviations of Continuous Variables

\begin{tabular}{lrrrrc}
\hline & \multicolumn{1}{l}{ n } & \multicolumn{1}{l}{ M } & \multicolumn{2}{l}{ Min } & Max \\
\hline Age & 69 & 22.55 & 6.14 & 18.00 & 59.00 \\
College Experience (Years) & 69 & 3.21 & 1.83 & 0.00 & 11.00 \\
Number Correct & 72 & 8.76 & 2.88 & 1.00 & 12.00
\end{tabular}


Table 3 Means and Standard Deviations for the Number of Correct Responses by Gender and Group

\begin{tabular}{lcccccc}
\hline & \multicolumn{2}{c}{ Low Error } & \multicolumn{2}{c}{ Higher Error } & \multicolumn{2}{c}{ Total } \\
& $\mathrm{n}$ & Mean & $\mathrm{n}$ & Mean & $\mathrm{n}$ & Mean \\
\hline Sound No Caption & 11 & $10.45^{\mathrm{a}}$ & 13 & $8.85^{\mathrm{b}, \mathrm{y}}$ & 24 & 9.58 \\
& & $(1.13)$ & & $(1.68)$ & & $(1.64)$ \\
Sound and Caption & 15 & 10.07 & 11 & $9.00^{\mathrm{y}}$ & 26 & 9.62 \\
& & $(1.10)$ & & $(2.90)$ & & $(2.08)$ \\
Caption No Sound & 13 & $9.69^{\mathrm{a}}$ & 9 & $2.78^{\mathrm{b}, \mathrm{x}}$ & 22 & 6.86 \\
& & $(21.45)$ & & $(1.72)$ & & $(3.80)$ \\
Total & 39 & 10.05 & 33 & 7.24 & 72 & 8.76 \\
& & $(1.23)$ & & $(3.48)$ & & $(2.88)$
\end{tabular}

Note. Standard deviations are shown in parenthesis below means. ${ }^{\mathrm{ab}}$ rows with different superscripts differed significantly, ${ }^{\mathrm{xy}}$ columns with different superscripts differed significantly

\section{Data Analysis}

A 2 (error: low vs. high) x 3 (caption: sound no caption vs. sound and caption vs. caption no sound) two-way analysis of variance (ANOVA) was conducted to examine the effect of error group and caption group on the number of correct responses.

There was a statistically significant interaction between error group and caption group, $F$ $(2,66)=19.69, p<.001$, partial eta squared $=.374$.

Simple main effects analyses revealed that participants in the low error group who watched the video with caption but no sound answered a significantly greater number of questions correctly $(M=9.69, S D=1.44)$ than participants in the high error group who watched the video with caption but no sound $(M=2.78, S D=1.72), p<.001$ (Figure 1). In addition, participants in the lower error group who watched the video with sound but no caption answered a significantly greater number of questions correctly $(M=10.45, S D=1.13)$ than participants in the high error group who watched the video with sound but no caption $(M=8.85, S D=1.68)$.

Simple main effects analyses also revealed that participants in the high error group who watched the video with caption but no sound answered a significantly fewer number of questions correctly $(M=2.78, S D=1.72)$ than participants in the high error group who watched the video with sound and caption $(M=9.00, S D=2.90)$ and participants in the high error group who watched the video with sound but no captions $(M=8.85, S D=1.68)$ (Figure 2$)$. 


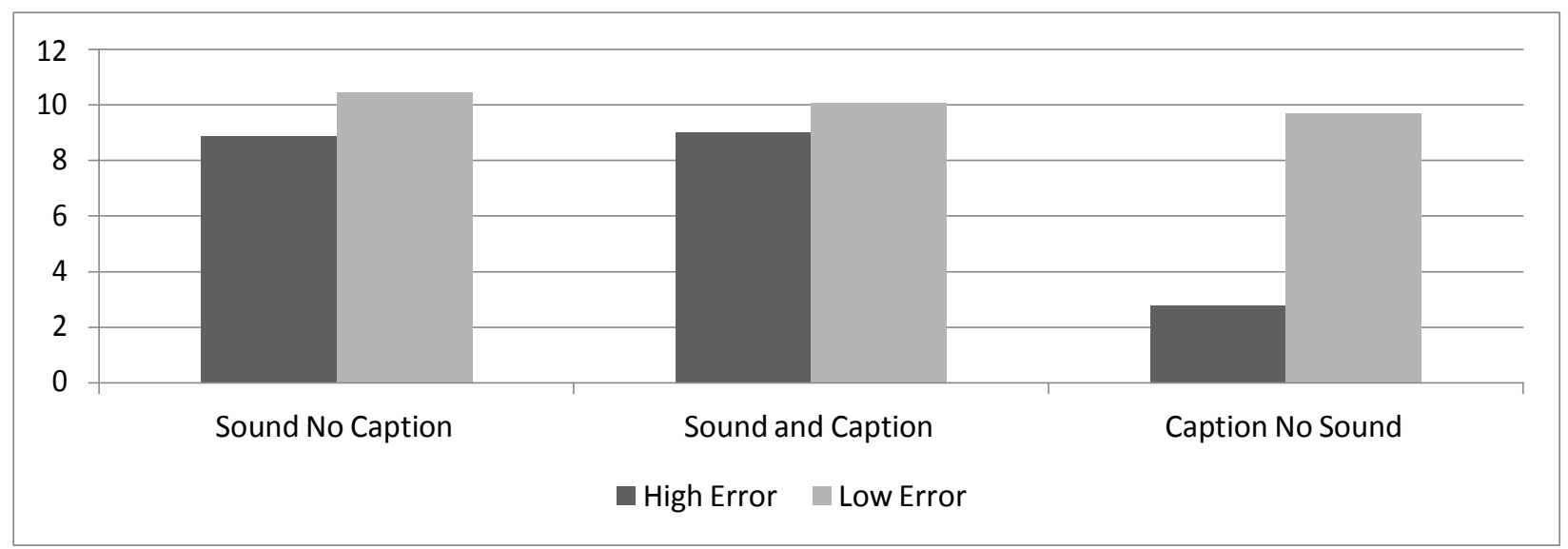

Figure 1: Comparison of comprehension errors between high and low captioning error

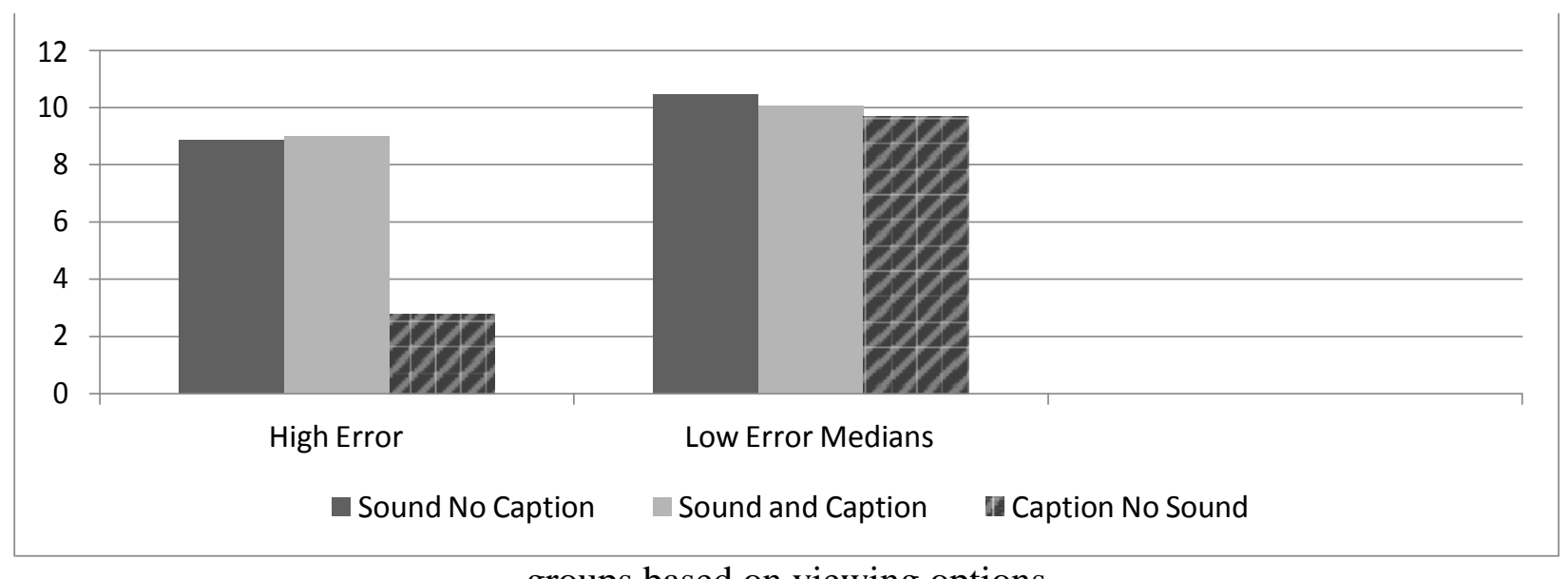

groups based on viewing options

Figure 2: Comparison comprehension low error frequency versus high error frequency based on viewing option

For the low error group, the three caption groups did not significantly differ in total correct scores. For the high error group, the caption no sounds group had significantly fewer correct scores than the sound no caption and the sound and caption groups (Figures 1 \& 2).

\section{Limitations and Future Directions}

This study was foundational research into the effectiveness of web-based automatic captioning for successful adult readers. Limitations to the study included a predominantly female participant group, stemming primarily from the make-up of the university's student body. Another limitation of the study was the use of successful readers. Future research should include the use of automatic captioning with struggling readers such as school-age deaf and hard of hearing students who demonstrate a variety of reading levels and who would typically be exposed to educational content similar to the content presented in this study. The extent to which such readers struggle with problematic automatic captioning should also be evaluated. While this 
research explored the abilities of individuals with native English reading experiences, future research should also include participants with limited English abilities to determine whether even accurate automatic captioning is problematic and the extent to which it is troublesome.

\section{Discussion}

There are several points of discussion based on the results of the study. Overall, the results indicated that the number of questions participants were able to answer correctly did not depend only on the type of error or caption group in which they were placed, they also depended on the interaction between the two types of groups. Participants in the high error group who watched a video with caption but no sound answered a significantly fewer number of questions correctly than participants in the high error group who watched a video with sound but no caption or both sound and caption.

The results clearly demonstrate that when auto-captions are presented accurately, regardless of whether sound is present, typical adult readers are able to comprehend the messages being delivered via text. Conversely, as assumed, when automatic-captions are presented inaccurately, containing significant numbers of errors, and when no audio content is available, even hearing, college-educated adult readers are unable to comprehend the messages being delivered. These results are significant in that participants in this study all have reading experience at the postsecondary level and were unable to accurately perceive automaticcaptioned messages with high errors that were delivered without sound. If adult readers with the ability to interact with print at the college level are unable to successfully navigate such automatic-captioned content, expecting school-age students who are deaf or hard of hearing with varied reading abilities to perform any better would be inappropriate as caption comprehension is highly and positively correlated with grade level (Lewis \& Jackson, 2001).

Captioning is critical for video to be accessible to students who are deaf or hard of hearing. However, the focus must be more than simply putting text on a screen, as appropriate captioning is imperative for successful comprehension. YouTube Teacher was created to help K12 teachers use educational video in their classrooms to support learning and engage and inspire learners. YouTube for Schools allows schools that opt-in to access thousands of educational videos (Buzzetto-More, 2014). Clearly the use of multimedia and the presentation of verbal and visual information will continue to be a common and recommended model of instruction in classrooms. Teachers need to become adept at using captioning accurately. As indicated earlier, teachers can manually caption their videos using several of the tools available in YouTube's video manager.

Accurate captioning is equally important for access at the university level. Faculty are using and recommending the use of online videos such as those found on YouTube (Moran, Seaman, \& Tinti-Kane, 2011; Tan \& Pearce, 2011). Betts, Cohen, Veit, Alphin, Broadus and Allen (2014) identified inaccessibility to videos and voice-over PowerPoint Presentations because they do not have captions as one of the greatest challenges for an online student with a hearing loss. Such recommendations carry the weight of assuring that videos for courses are accessible to all students. Relying on the automatic captioning feature of YouTube will be insufficient to provide student users who require the captioned text for comprehension. 
Captioning is vital but needs to be studied. We are not suggesting getting rid of it—only finding out what works best. Further discussion regarding the automatic captioning of web-based video content should center on several issues, including mandating which web-based content should be permitted to employ automatic captioning features and how to improve upon the infrastructure of automatic captioning and speech recognition platforms.

\section{References}

Asuncion, J. V., Budd, J., Fichten, C. S., Nguyen, M. N., Barile, M., \& Amsel, R. (2012). Social media use by students with disabilities. Academic Exchange Quarterly, 16(1), 30-35.

Betts, K., Cohen, A.H., Veit, D.P., Alphin, H.C., Broadus, C., \& Allen, D. (2014). Strategies to increase online student success for students with disabilities. Journal of Asynchronous Learning, 17(3), 50-65.

Barton, F., Bradbrook, G., \& Broome, G. (2015). Digital accessibility: A report for citizens online. Edinburgh, Scotland.

Buzzetto-More, N. (2015) An examination of undergraduate student's perceptions and predilections of the use of YouTube in the teaching and learning process, Interdisciplinary Journal of e-learning and learning objects. Retrieved from https://www.questia.com/library/journal/1G1-418467094/an-examination-ofundergraduate-student-s-perceptions.

Cambra, C., Silvestre, N., \& Leal, A. (2009). Comprehension of television messages by deaf students at various levels of education. American Annals of the Deaf, 153(5), 425-434.

Dahbi, M. (2004). English and Arabic after 9/11. The Modern Language Journal, 88(4), 628630.

Fichten, C. S., Asuncion, J., \& Scapin, R. (2014). Digital Technology, Learning, and Postsecondary Students with Disabilities: Where We've Been and Where We're Going. Journal of Postsecondary Education and Disability, 27(4), 369-379.

Google. (2015). Auto captioning [YouTube Help: Edit videos \& setting: Caption]. Retrieved from https://support.Google.com/YouTube /answer/3038280?hl=en

Jensema, C. \& McCann, R. (1996). Presentation Speed and Vocabulary in Closed Captioned Television. American Annals of the Deaf, 141(4).

Johnson, A. (2014). Video Captioning Policy and Compliance at the University of Minnesota Duluth. Unpublished Master’s Thesis, University of Minnesota - Duluth, USA.

Johnson-Glenberg, M. (2000). Training reading comprehension in adequate decoders/poor comprehenders: Verbal and visual strategies. Journal of Educational Psychology, 92(4), 772-782. 
Lewis, M. \& Jackson, D. (2001). Television literacy: Comprehension of program content using closed captions for the Deaf. Journal of Deaf Studies and Deaf Education, 43-53.

Moran, M., Seaman, J., \& Tinti-Kane, H. (2011). Teaching, Learning, and Sharing: How Today's Higher Education Faculty Use Social Media. Babson Survey Research Group.

Paas, F., \& van Merriënboer, J. J. G. (1994a). Instructional control of cognitive load in the training of complex cognitive tasks. Educational Psychology Review, 6, 51-71.

Paas, F., Tuovinen, J. E., Tabbers, H., \& Van Gerven, P. W. (2003). Cognitive load measurement as a means to advance cognitive load theory. Educational psychologist, 38(1), 63-71.

Ruan, X. (2015). The Effect of Caption Modes on EFL Students' Video Comprehension. Journal of Language Teaching and Research, 6, 2, 397-404.

Sadoski, M., \& Paivio, A. (2004) A dual-coding theoretical model of reading. In R. B. Ruddell \& N. N. Unrau (Eds.), Theoretical models and processes of reading (5th ed., pp. 13291362). Newark, DE: International Reading Association.

Seale, J., Georgeson, J., Mamas, C., \& Swain, J. (2015). Not the right kind of 'digital capital'? An examination of the complex relationship between disabled students, their technologies and higher education institutions. Computers \& Education, 82, 118-128.

Stinson, M. S., \& Stevenson, S. (2013). Effects of expanded and standard captions on deaf college students' comprehension of educational videos, American Annals of the Deaf, 158(4), 453-467.

Tan, E., \& Pearce, N. (2012). Open education videos in the classroom: exploring the opportunities and barriers to the use of YouTube in teaching introductory sociology. Research in Learning Technology, 19.

Texas Educational Agency (2010). Chapter 112. Texas Essential Knowledge and Skills for Science, Subchapter B. Middle School. Retrieved from http://ritter.tea.state.tx.us/rules/tac/chapter112/ch112b.html\#112.20

Winke, P., Gass, S., \& Sydorenko, T. (2010). The effects of captioning videos used for foreign language listening activities. Language Learning \& Technology, 14(1), 65-86.

3 Play Media (2014). Future of accessibility and video captions according to google and YouTube. Retrieved from http://www.3playmedia.com/2014/01/10/future-accessibilityvideo-captions-according-google-youtube/ 


\section{APPENDIX A: VIDEOS \& VIEWING CONDITIONS \\ Group \\ \# \\ Description \\ VIDEO ONE LOCATION: http://www.YouT ube .com/watch?v=B1AXbpYndGc}

1 Video 1(low error) sound no caption

2 Video 1(low error) sound and caption

3 Video 1(low error) caption no sound

*VIDEO TWO LOCATION: http://www.You Tube .com/watch?v=RJ0JCg3S7xQ

4 Video 2(medium error) sound no caption

5 Video 2(medium error) sound and caption

6 Video 2(medium error) caption no sound

VIDEO THREE LOCATION: http://www.Yo uTube .com/watch?v=tDnawSj64jg

7 video 3(high error) sound no caption

8 Video 3(high error) sound and caption

9 Video 3(high error) caption no sound

*No participants viewed this group of videos. Originally, the medium-error videos were to be viewed. When it became apparent that the number of participants were going to be limited, the authors chose to focus attention on the high-error and low-error videos. 
APPENDIX B: VIDEO VIEWING QUESTIONNAIRE EXAMPLE Video Questionnaire: GROUP 1

"Naked Science: Birth of the Solar System"

http://www.youtube.com/watch?v=B1AXbpYndGc

1. A dense clump of water formed what?

2. When a star reaches 18 million degrees Fahrenheit what kicks in?

3. When was our star(the sun) born?

4. What fuses together to form helium?

5. What is the first type of light made by our sun?

6. Was the solar system's birth peaceful?

7. Where was the sun born?

8. An entire universe was supposed to be created from what?

9. A big explosion, that caused the creation of the universe, is known as?

10. There are how many naturally occurring chemical elements?

11. What are two elements that planets are made of?

12. Hydrogen and Helium fuse to make what? 
Gender: M F $\quad \mathrm{T} \quad$ Is English your first language? $\mathrm{Y} \quad \mathrm{N}$ Age:

\# of years college experience? _ _ Hearing status: Hearing Deaf Hard of Hearing

YouTube Automatic captioning Participant \# 